\title{
Website Modeling and Website Generation
}

\author{
Bernhard Thalheim ${ }^{1}$, Klaus-Dieter Schewe ${ }^{2}$, Irina Romalis ${ }^{3}$, \\ Thomas Raak ${ }^{1,3}$, and Gunar Fiedler ${ }^{1}$ \\ 1 University Kiel, Institute of Computer Science, Olshausenstrasse 40, 24098 Kiel, \\ Germany \\ 2 Massey University, Private Bag 11 222, Palmerston North, New Zealand, \\ 3 Cottbus University of Technology, Computer Science Insitute, PostBox 101344, \\ 03013 Cottbus, Germany
}

\section{Website Specification}

Websites become more often based on data warehouse architectures. Our group uses the website modeling language SiteLang [TD01]. This language has reached a maturity that allows simple application and goes far beyond what other approaches can handle: It is powerful, consistent, has a well-developed theory fundament Tha00a Tha00b, does not allow to create confusing specifications, uses a powerful methodology [ST00 Tha03 applied in three dozen projects, and has a playout and layout specification supporting database-backed generation of websites.

\section{Application in Projects Resulting in Installation of Large or Huge Websites}

The approach presented in this paper is based on the experience the Cottbus Information Services team gained during successful development of 36 large or huge websites (partially with more than 15.000 pages), e.g., 17 large municipality websites (information and community sites), 5 service sites, 2 large learning sites, 4 group and community sites, 1 e-government sites, 1 huge B2B site, and 1 cable-net backed, set-top-box based TV-internet platform.

\section{Automatic Generation, Orchestration, and Maintenance of Websites}

The SiteLang specification can be developed by an editor that has been developed for support of our website projects and is applied for generation of websites. The storyboard editor uses a four window representation representing scenarios, dialogs and dialog steps, database and content base, and story properties. The website developer uses the editor backed by a database that contains the website structure and functionality. The editor supports integration of content obtained from other content systems and databases. This information is used for automatic generation of the website whenever content, structuring, and functionality are changed. Website developers do no longer develop XML document hills. Instead, the XML documents are generated by the system. 


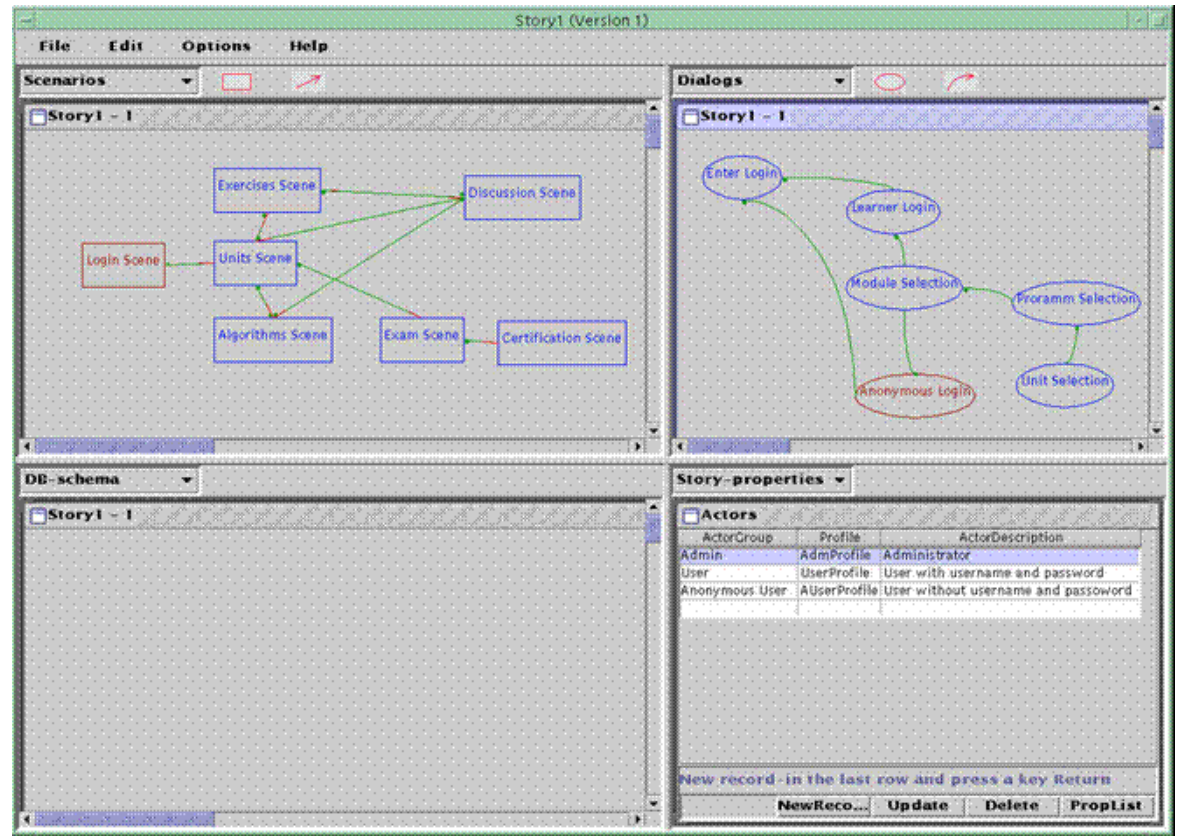

Fig. 1. A Screeenshot from the Storyboard Editor

\section{References}

[ST00] K.-D. Schewe and B. Thalheim. Tutorial Notes. ER 2000, 2000.

[TD01] B. Thalheim and A. Düsterhöft. Sitelang: Conceptual modeling of internet sites. In Proc. ER'01, LNCS 2224, Springer, pages 179-192, 2001.

[Tha00a] B. Thalheim. Entity-relationship modeling - Foundations of database technology. Springer, Berlin, 2000. See also http://www.informatik.tu-cottbus.de/ thalheim/HERM.htm.

[Tha00b] B. Thalheim. Readings in fundamentals of interaction in information systems. Reprint, BTU-Cottbus, accessible through http://www.informatik.tucottbus.de/ thalheim, Collection of papers by C. Binder, W. Clauß, A. Düsterhöft, T. Feyer, T. Gutacker, B. Heinze, J. Lewerenz, M. Roll, B. Schewe, K.-D. Schewe, K. Seelig, S. Srinivasa, B. Thalheim, 2000.

[Tha03] B. Thalheim. Co-design of structuring, funtionality, distribution, and interactivity of large information systems. Coumputer Science Reports 15/03, Cottbus University of Technology, Computer Science Institute, 2003. 